\title{
A Time Dependent Model for Image Denoising
}

\author{
Santosh Kumar, Mohammad Kalimuddin Ahmad \\ Department of Mathematics Aligarh Muslim University, Aligarh, India \\ Email: skykumar87@gmail.com, ahmad_kalimuddin@yahoo.co.in
}

Received 27 January 2015; accepted 16 February 2015; published 25 February 2015

Copyright $@ 2015$ by authors and Scientific Research Publishing Inc.

This work is licensed under the Creative Commons Attribution International License (CC BY).

http://creativecommons.org/licenses/by/4.0/

(c) (1) Open Access

\section{Abstract}

In this paper, we propose a new time dependent model for solving total variation (TV) minimization problem in image denoising. The main idea is to apply a priori smoothness on the solution image. This is a constrained optimization type of numerical algorithm for removing noise from images. The constraints are imposed using Lagrange's multipliers and the solution is obtained using the gradient projection method. 1D and 2D numerical experimental results by explicit numerical schemes are discussed.

\section{Keywords}

Total Variation, Image Denoising, Signal Denoising

\section{Introduction}

In many image processing problems, a denoising step is required to remove noise or spurious details from corrupted images. The presence of noise in images is unavoidable. It may be introduced at the stage of image formation like image recording, image transmission, etc. These random distortions make it difficult to perform any required image analysis. For example, the feature oriented enhancement introduced in [1] is very effective in restoring blurry images, but it can be "frozen" by an oscillatory noise component. Even a small amount of noise is harmful when high accuracy is required, especially in case of medical images.

In practice, to estimate a true signal in noise, the most frequently used methods are based on the least squares criteria. This procedure is $L^{2}$-norm dependent. $L^{2}$-norm based regularization is known to remove high frequency components in denoised images and make them appear smooth.

Most of the classical image deblurring or denoising techniques, due to linear and global approach, are 
contaminated by Gibb's phenomenon resulting into smearing near edges. In order to preserve edges Rudin et al. [2] [3] introduced total variation (TV) norm models based on variational approach. TV norms are essentially $L^{1}$ norms derivatives, hence $L^{1}$ estimation procedures are more appropriate for the subject of image restoration. For more details we refer to [1] [4]-[7].

In this paper we present a new time dependent model constructed by evolving the Euler-Lagrange equations of the optimization problem. We propose to apply priori smoothness on the solution image and then denoise it by minimizing the total variation norm of the estimated solution. We have tested our algorithm on various types of signals and images and found our model (11) better than previously known model (10). To quantify results, the experimental values in terms of PSNR are given in Tables 1-3.

\section{Image Denoising Models}

Formation of a noisy image is typically modeled as

$$
u_{0}(x, y)=u(x, y)+n(x, y),
$$

where $u(x, y)$ denote the desired clean image, $u_{0}(x, y)$ denote the pixel values of a noisy image for $x, y \in \Omega, \Omega$ is a bounded open subset of $\mathbb{R}^{2}$ and $n(x, y)$ is additive white noise assumed to be close to Gaussian. The values $n(i, j)$ of $n$ at the pixels $(i, j)$ are independent random variables, each with a Gaussian distribution of zero mean and variance $\sigma^{2}$.

We wish to reconstruct $u$ from $u_{0}$. Most conventional variational methods involve a least squares $L^{2}$ fit because this leads to linear equations. The first attempt along these lines was made by Phillips [8] and later refined by Twomey et al. [9] [10] in one-dimensional case. In two dimensional continuous framework their constrained minimization problem is,

$$
\operatorname{minimize} \int_{\Omega}\left(u_{x x}+u_{y y}\right)^{2} \mathrm{~d} x \mathrm{~d} y,
$$

subject to constraints involving the mean

$$
\int_{\Omega} u \mathrm{~d} x \mathrm{~d} y=\int_{\Omega} u_{0} \mathrm{~d} x \mathrm{~d} y
$$

and standard deviation

$$
\int_{\Omega}\left(u-u_{0}\right)^{2} \mathrm{~d} x \mathrm{~d} y=\sigma^{2} .
$$

The resulting linear system is now easy to solve using modern numerical techniques.

The total variation based image denoising model, which is based on the constrained minimization problem appeared in [2], is as follows:

$$
\operatorname{minimize} \int_{\Omega}|\nabla u| \mathrm{d} x \mathrm{~d} y=\int_{\Omega} \sqrt{u_{x}^{2}+u_{y}^{2}} \mathrm{~d} x \mathrm{~d} y
$$

subject to constraints

$$
\int_{\Omega} u \mathrm{~d} x \mathrm{~d} y=\int_{\Omega} u_{0} \mathrm{~d} x \mathrm{~d} y
$$

and

$$
\int_{\Omega} \frac{1}{2}\left(u-u_{0}\right)^{2} \mathrm{~d} x \mathrm{~d} y=\sigma^{2}
$$

The first constraint corresponds to the assumption that the noise has zero mean, and the second constraint uses a priori information that the standard deviation of the noise $n(x, y)$ is $\sigma$.

The Euler-Lagrange equation is given by,

$$
0=\nabla \cdot\left(\frac{\nabla u}{|\nabla u|}\right)-\lambda_{1}-\lambda_{2}\left(u-u_{0}\right),
$$


in $\Omega$, with $\frac{\partial u}{\partial n}=0$ on the boundary of the domain.

Since (8) is not well defined at points where $\nabla u=0$, due to the presence of the term $\frac{1}{|\nabla u|}$, it is common to slightly perturb the TV algorithm to become

$$
\int_{\Omega}|\nabla u|_{\beta} \mathrm{d} x \mathrm{~d} y=\int_{\Omega} \sqrt{u_{x}^{2}+u_{y}^{2}+\beta} \mathrm{d} x \mathrm{~d} y,
$$

where $\beta$ is a small positive parameter [11].

The solution procedure uses a parabolic equation with time as an evolution parameter, or equivalently, the gradient descent method. This means that we solve

$$
u_{t}=\nabla \cdot\left(\frac{\nabla u}{|\nabla u|}\right)-\lambda\left(u-u_{0}\right)
$$

for $t>0, x, y \in \Omega$ with $u(x, y, 0)$ given as initial data and $\frac{\partial u}{\partial n}=0$ on the boundary of the domain.

Applying a priori smoothness on the solution image, our new time dependent model becomes,

$$
u_{t}=\nabla \cdot\left(\frac{\nabla G_{\sigma} * u}{\left|\nabla G_{\sigma} * u\right|}\right)-\lambda\left(G_{\sigma} * u-u_{0}\right),
$$

for $t>0, x, y \in \Omega$ with $u(x, y, 0)$ given as initial data and $\frac{\partial u}{\partial n}=0$ on the boundary of the domain. It should be noticed that (11) only replaces $u$ in (10) by its estimate $G_{\sigma} * u$.

Witkin [12] noticed that the convolution of the signal with Gaussians at each scale was equivalent to solving the heat equation with the signal as initial datum. The term $\left(G_{\sigma} * \nabla u\right)(x, y, t)=\left(\nabla G_{\sigma} * u\right)(x, y, t)$, which appears inside the divergence term of (11), is simply the gradient of the solution at time $\sigma$ of the heat equation with $u(x, y, 0)$ as initial datum. In order to preserve the notion of scale in the gradient estimate, it is convenient that this kernel $G_{\sigma}$ depends on a scale parameter [13]. In fact, the function $G_{\sigma}$ can be considered as "low-pass filter" or any smoothing kernel, i.e., a denoising technique is used before solving the nonlinear diffusion problem [14] [15].

The first constraint (8) is dropped because it is automatically enforced by the evolution procedure, i.e., the mean of $u(x, y, 0)$ is the same as that of $u_{0}(x, y)$. As $t$ increases, a denoised version of image is realised.

To compute $\lambda(t)$, we multiply (10) by $\left(u-u_{0}\right)$ and integrate by parts over $\Omega$. If steady state has been reached, the left side of (10) vanishes. We then have,

$$
\lambda=-\frac{1}{2 \sigma^{2}} \int_{\Omega}\left[|\nabla u|-\left(\frac{\left(u_{0}\right)_{x} u_{x}}{|\nabla u|}+\frac{\left(u_{0}\right)_{y} u_{y}}{|\nabla u|}\right)\right] \mathrm{d} x \mathrm{~d} y .
$$

This gives us a dynamic value $\lambda(t)$, which appears to converge as $t \rightarrow \infty$. The theoretical justification for this approach comes from the fact that it is merely the gradient projection method of Rosen [16].

We still write $G_{\sigma} * u$ as $u$. Let $u_{i j}^{n}$ be the approximation to the value $u\left(x_{i}, y_{j}, t_{n}\right)$, where

$$
\begin{gathered}
x_{i}=i \Delta x, \quad y_{j}=j \Delta x, \quad i, j=1,2, \cdots, N, \\
N \Delta x=1, t_{n}=n \Delta t, n=0,1, \cdots, \\
u_{i j}^{n}=u\left(x_{i}, y_{j}, t_{n}\right), \\
u_{i j}^{0}=u_{0}(i \Delta x, j \Delta x)+\sigma \phi(i \Delta x, j \Delta x) .
\end{gathered}
$$

The modified initial data are chosen so that the constraints are satisfied initially, i.e., $\phi$ has mean zero and $L^{2}$ norm one. 
The explicit partial derivatives of model (10) and model (11) can be expressed as:

$$
u_{t}=\frac{u_{x x}\left(u_{y}^{2}+\beta\right)-2 u_{x y} u_{x} u_{y}+u_{y y}\left(u_{x}^{2}+\beta\right)}{\left(u_{x}^{2}+u_{y}^{2}+\beta\right)^{\frac{3}{2}}}-\lambda\left(u-u_{0}\right) .
$$

We define the derivative terms as,

$$
\begin{gathered}
u_{i j}^{x}=\frac{u_{i+1, j}^{n}-u_{i-1, j}^{n}}{2 \Delta x} ; \quad u_{i j}^{y}=\frac{u_{i, j+1}^{n}-u_{i, j-1}^{n}}{2 \Delta x} ; \\
u_{i j}^{x x}=\frac{u_{i+1, j}^{n}-2 u_{i, j}^{n}+u_{i-1, j}^{n}}{\Delta x^{2}} ; \quad u_{i j}^{y y}=\frac{u_{i, j+1}^{n}-2 u_{i, j}^{n}+u_{i, j-1}^{n}}{\Delta x^{2}} ; \\
u_{i j}^{x y}=\frac{u_{i+1, j+1}^{n}-u_{i-1, j+1}^{n}-u_{i+1, j-1}^{n}+u_{i-1, j-1}^{n}}{4 \Delta x \Delta x} ; u_{i j}^{t}=\frac{u_{i, j}^{n+1}-u_{i, j}^{n}}{\Delta t} .
\end{gathered}
$$

We let,

$$
r_{i j}^{n}=u_{i j}^{x x}\left(\left(u_{i j}^{y}\right)^{2}+\beta\right)-2 u_{i j}^{x y} u_{i j}^{x} u_{i j}^{y}+u_{i j}^{y y}\left(\left(u_{i j}^{x}\right)^{2}+\beta\right)
$$

and

$$
p_{i j}^{n}=\left(\left(u_{i j}^{x}\right)^{2}+\left(u_{i j}^{y}\right)^{2}+\beta\right)^{\frac{3}{2}} .
$$

Then (14) reads as follows:

$$
u_{i j}^{t}=\frac{r_{i j}^{n}}{p_{i j}^{n}}-\lambda\left(u_{i j}^{n}-u_{0}(i \Delta x, j \Delta x)\right)
$$

with boundary conditions

$$
\begin{aligned}
& u_{i, N}^{n}=u_{i, N-1}^{n}, \quad u_{N, j}^{n}=u_{N-1, j}^{n}, \\
& u_{1, j}^{n}=u_{2, j}^{n}, \quad u_{i, 1}^{n}=u_{i, 2}^{n} .
\end{aligned}
$$

The explicit method is stable and convergent for $\frac{\Delta t}{\Delta x^{2}} \leq 0.5$, see [17].

\section{Time Dependent Model for 1D}

The 2D model described before is more regular than the corresponding $1 \mathrm{D}$ model because the $1 \mathrm{D}$ original optimization problem is barely convex. For the sake of understanding the numerical behavior of our schemes, we also discuss the 1D model. The Euler-Lagrange equation in the 1D case reads as follows:

$$
0=\left(\frac{u_{x}}{\left|u_{x}\right|}\right)_{x}-\lambda_{1}-\lambda_{2}\left(u-u_{0}\right)
$$

This equation can be written either as

$$
0=\left(\frac{u_{x}}{\left|u_{x}\right|_{\beta}}\right)_{x}-\lambda_{1}-\lambda_{2}\left(u-u_{0}\right),
$$

using the small regularizing parameter $\beta>0$ introduced in [18], or

$$
0=\delta\left(u_{x}\right) u_{x x}-\lambda_{1}-\lambda_{2}\left(u-u_{0}\right) \text {, }
$$

using the $\delta$-function.

Our model in $1 \mathrm{D}$ will be 


$$
u_{t}=\frac{\beta}{\left(\beta+u_{x}^{2}\right)^{\frac{3}{2}}} u_{x x}-\lambda\left(u-u_{0}\right),
$$

where $\beta>0$ is small regularizing parameter. The parameter $\beta>0$ in this model is estimated from the local amount of noise. We have found for our model, through our numerical experiments in $1 \mathrm{D}$, that $\beta$ can be estimated as the standard deviation of the noise.

We can also state our model in terms of the $\delta$ function as

$$
u_{t}=\delta\left(u_{x}\right) u_{x x}-\lambda\left(u-u_{0}\right) .
$$

In this paper, we approximate $\delta$, see the reference [18], by

$$
\delta(k) \approx \beta \cdot\left(k^{2}+\beta\right)^{-\frac{3}{2}} .
$$

These evolution models are initialized with the noisy signal $u_{0}$, homogeneous Neumann boundary conditions, and with a prescribed Lagrange multiplier for slightly noisy signals.

We have estimated $\lambda>0$ near the maximum value such that the explicit scheme is stable under appropriate CFL $\left(\frac{\Delta t}{\Delta x^{2}}<0.25\right)$ restrictions [18], provided $\beta$ is chosen to be the standard deviation of the noise.

The following is the explicit numerical scheme of model (22).

Let $u_{i}^{n}$ be the approximation to the value $u\left(x_{i}, t_{n}\right)$, where $x_{i}=i \Delta x$ and $t_{n}=n \Delta t, n \geq 1$. We define the derivative terms as,

$$
u_{x}=\frac{u_{i+1}^{n}-u_{i-1}^{n}}{2 \Delta x} ; u_{t}=\frac{u_{i}^{n+1}-u_{i}^{n}}{\Delta t} ; u_{x x}=\frac{u_{i+1}^{n}-2 u_{i}^{n}+u_{i-1}^{n}}{\Delta x^{2}} .
$$

We let,

$$
b_{i}=\frac{u_{i+1}^{n}-u_{i-1}^{n}}{2 \Delta x} .
$$

Then (22) reads as follows:

$$
u_{i}^{n+1}=u_{i}^{n}+\Delta t\left[\frac{\beta}{\left(\beta+b_{i}^{2}\right)^{\frac{3}{2}}} \frac{u_{i+1}^{n}-2 u_{i}^{n}+u_{i-1}^{n}}{\Delta x^{2}}\right]-\Delta t \lambda\left(u_{i}^{n}-u_{0}\left(x_{i}\right)\right) .
$$

\section{Numerical Experiments for 1D}

We, as an example, have taken 1D signals $u(x)=\exp (0.1 x) \cdot|\sin (x)|, x \in[0,25]$ and

$u(x)=|\sin (x)+\cos (x)|, x \in[0,25]$ given in Figure 1(a) and Figure 1(b) respectively. When Gaussian white noise is added to them, we get noisy signals.

In our test, we will use the signal to noise ratio (SNR) of the signal $u$ to measure the level of noise, defined as

$$
\mathrm{SNR}=\frac{\|u-\bar{u}\|_{L^{2}}}{\sigma}
$$

where $\bar{u}$ is the mean of the signal $u$, i.e., the ratio of the standard deviation of the signal over the standard deviation of the noise.

The standard deviation of noisy signals (given in Figure 1(c) and Figure 1(d)) are approximately $\sigma \approx 0.5$ and $\sigma \approx 0.04$ respectively whereas their SNR are 0.99 and 0.95 respectively.

We use $\beta=\sigma$ ( $\sigma$ is the standard deviation of the noise) and the Langrange multiplier $\lambda=0.005$ [18]. Figure 1(e) and Figure 1(f) represent the denoised signals after 80 iterations with SNR $\approx 1.1$ and 1.12 respectively. 


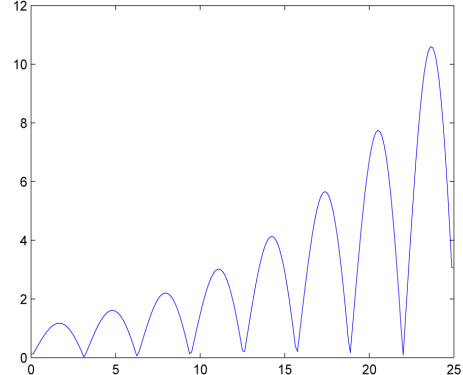

(a)

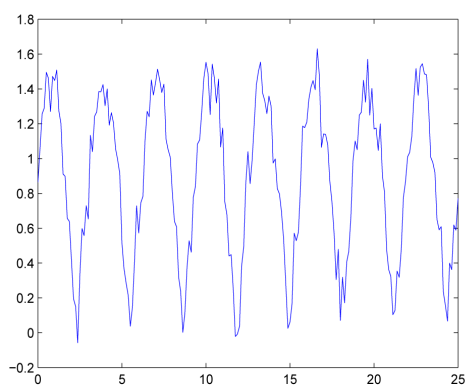

(d)

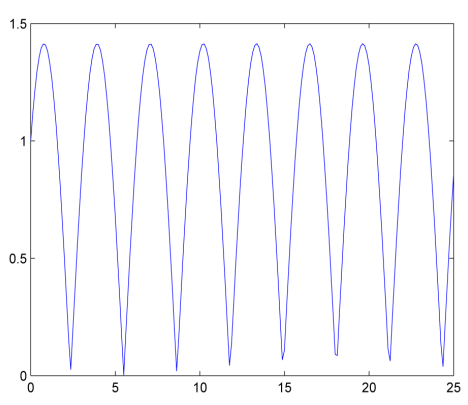

(b)

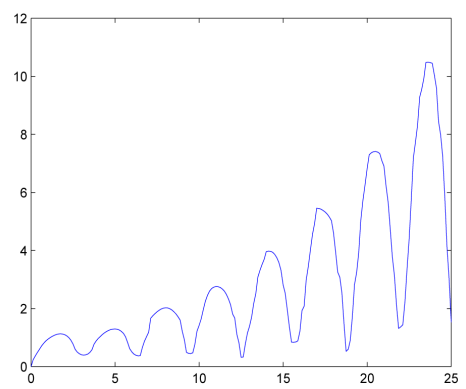

(e)

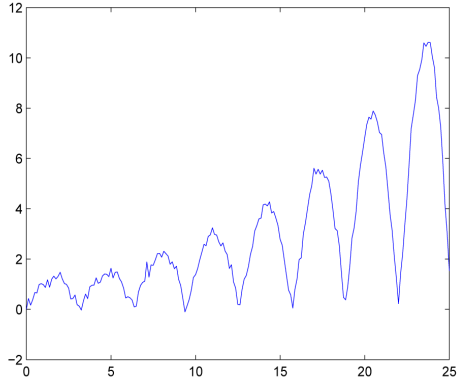

(c)

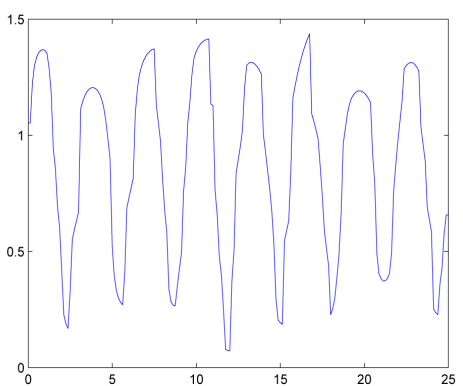

(f)

Figure 1. (a) (b) Original signals; (c) (d) Corresponding noisy signals; (e) (f) Corresponding denoised signals by model (22).

We have performed many other experiments on 1D signals obtaining similar results.

\section{Numerical Experiments for 2D}

In our tests, we use peak signal to noise ratio (PSNR) as a criteria for the quality of restoration. This quality is usually expressed in terms of the logarithmic decibel scale:

$$
\text { PSNR }=10 \log _{10}\left(\frac{R^{2}}{\frac{1}{m n} \sum_{i, j}^{n}\left(u(i, j)-u_{\text {new }}(i, j)\right)^{2}}\right),
$$

where $\left(u(i, j)-u_{\text {new }}(i, j)\right)$ are the differences of the pixel values between the original and denoised images and $R$ is the maximum fluctuation in the input image data.

When Gaussian white noise with mean zero and variance $\sigma^{2}$ is added to the original images, we get noisy images. In our experiment, we have considered the images corrupted with different levels of Gaussian noise. Figures 3(a)-(c), Figures 4(a)-(c) and Figures 5(a)-(c) contain noisy images with different levels of Gaussian noise. The results obtained by using models (10) and (11) are shown in Figures 3-5 and Tables 1-3. We have taken Lagrange multiplier $\lambda=0.85$ as was used in references [19] and [11]. We can choose $\beta=10^{-32}$ [11], the smallest positive machine number.

We have used three gray scale images, Goldhill $(256 \times 256)$, Rice $(256 \times 256)$ and Boat $(512 \times 512)$ shown in Figure 2 for our denoising experiments.

The values of PSNR obtained using model (11) given in Tables 1-3 are larger than that of using model (10) at the same iteration number. Thus based on PSNR values and also on human perception, we conclude that the model (11) gives better denoised images than that of model (10).

\section{Concluding Remarks}

We have presented a new time dependent model (11) to solve the nonlinear total variation problem for image denoising. The main idea is to apply a priori smoothness on the solution image. Nonlinear explicit schemes are 


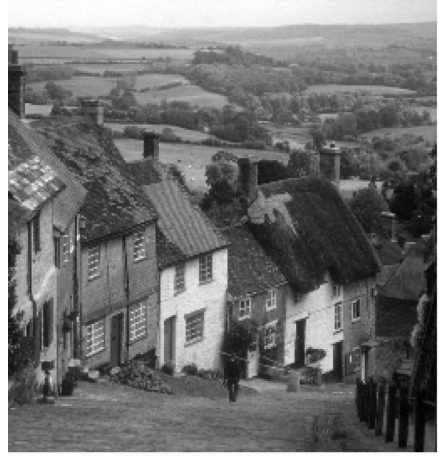

(a)

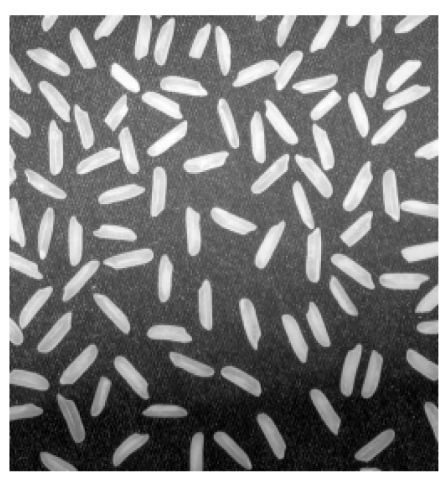

(b)

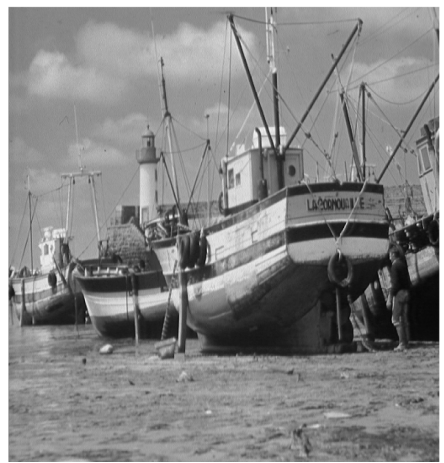

(c)

Figure 2. Original test images used for different experiments. (a) Goldhill: $256 \times 256$; (b) Rice: $256 \times 256$; (c) Boat: $512 \times$ 512.

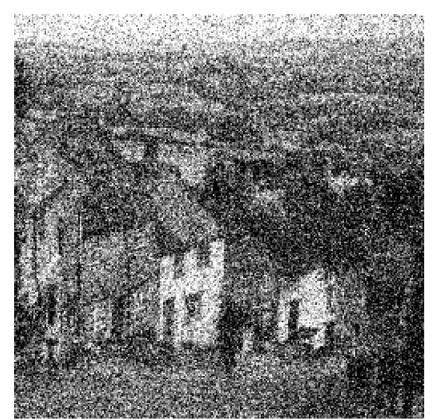

(a)

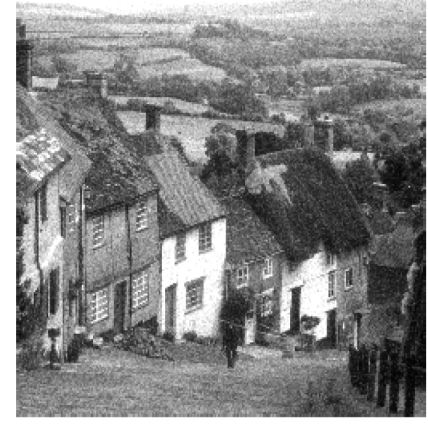

(d)

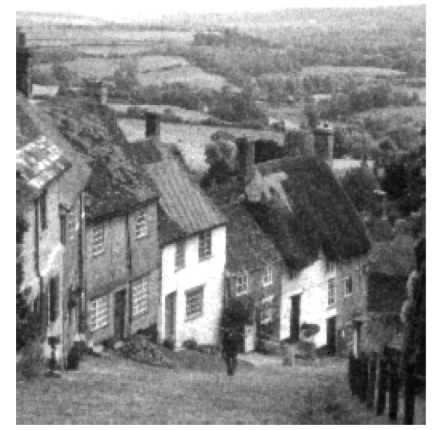

(g)

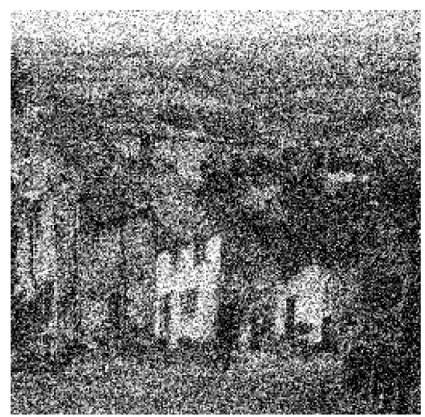

(b)

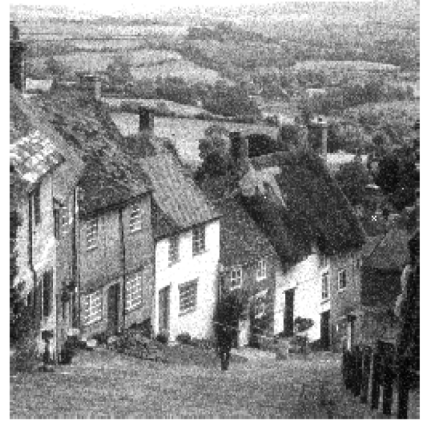

(e)

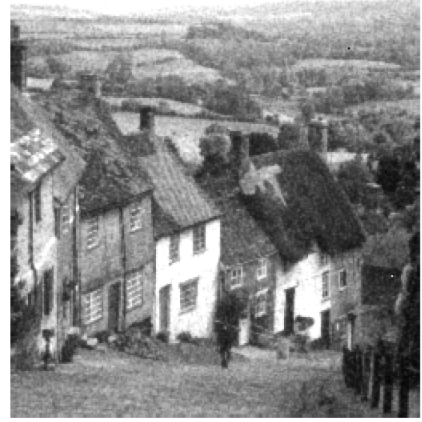

(h)

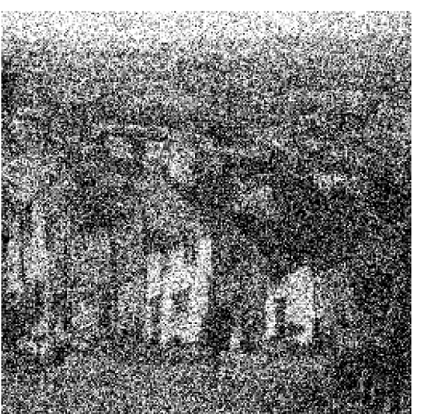

(c)

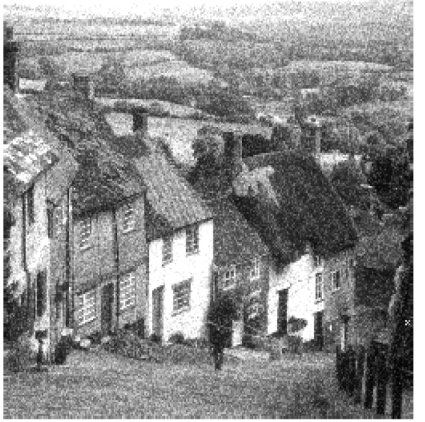

(f)

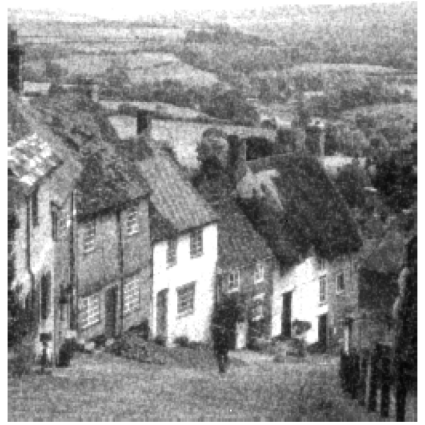

(i)

Figure 3. (Top row) Noisy Goldhill images with different levels of Gaussian noise (a)-(c), $\sigma^{2}=0.06,0.08$, 0.10, respectively; (Second row) (d)-(f) corresponding denoised images by model (10); (Third row) (g)-(i) by model (11). 


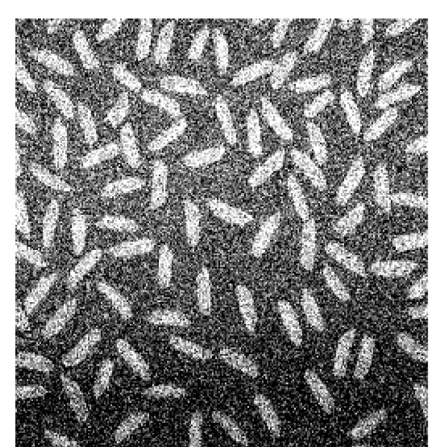

(a)

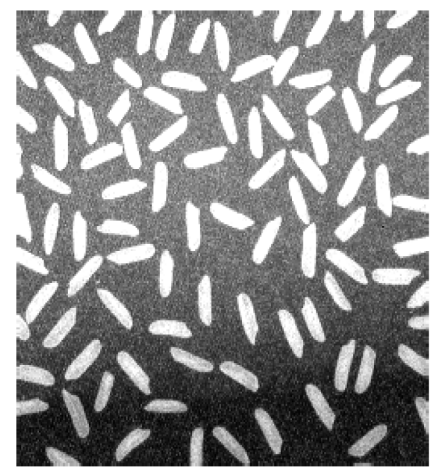

(d)

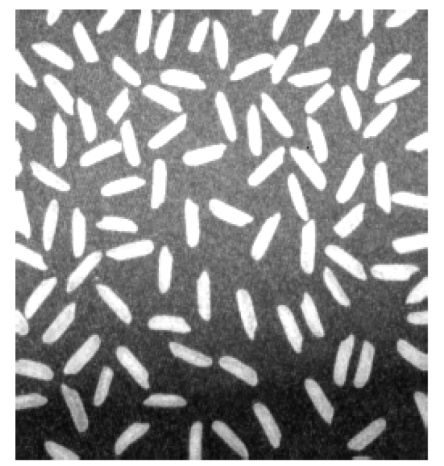

(g)

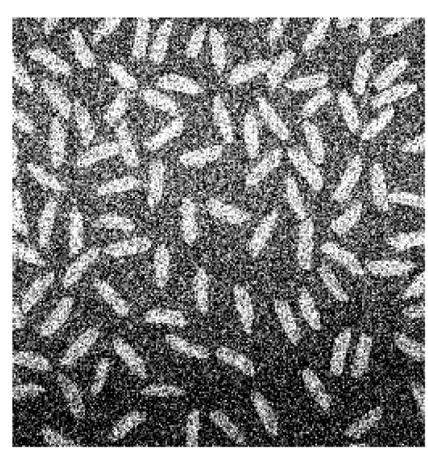

(b)

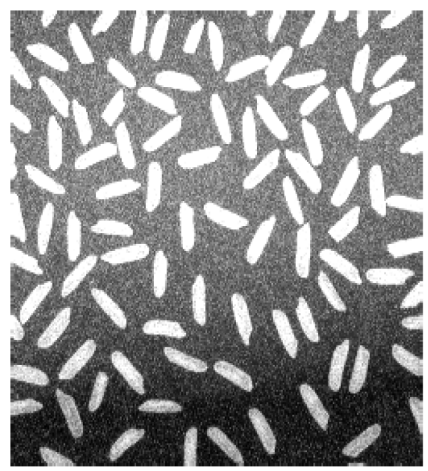

(e)

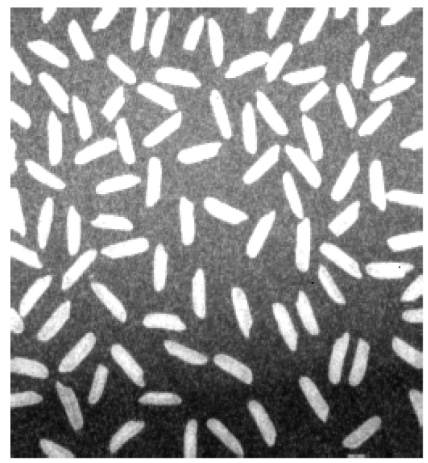

(h)

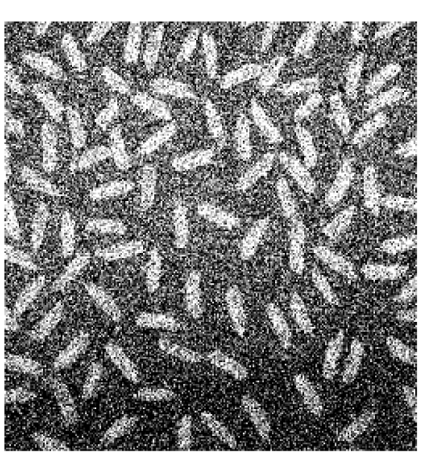

(c)

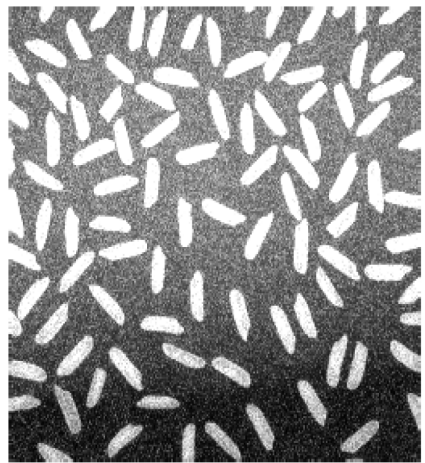

(f)

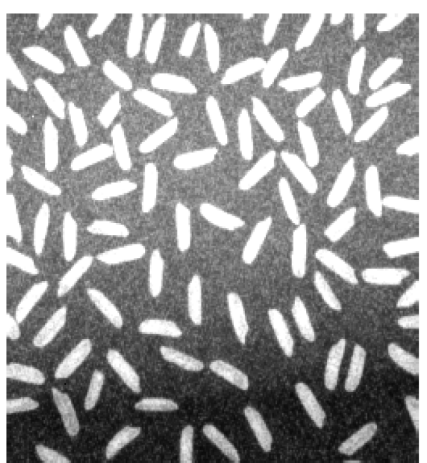

(i)

Figure 4. (Top row) Noisy Rice images with different levels of Gaussian noise (a)-(c); $\sigma^{2}=0.06,0.08,0.10$, respectively; (Second row) (d)-(f) corresponding denoised images by model (10); (Third row) (g)-(i) by model (11).

Table 1. Results obtained by using models (10) and (11) applied to the images in Figure 3 with three different levels of Gaussian noise $\left(\sigma^{2}=0.06,0.08\right.$ and 0.10$)$.

\begin{tabular}{cccccc}
\hline Images & PSNR & Images & PSNR & Images & PSNR \\
\hline & (Noisy images) & & (Model-10) & & (Model-11) \\
\hline Figure 3(a) & 13.18 & Figure 3(d) & 18.79 & Figure 3(g) & 19.30 \\
Figure 3(b) & 12.23 & Figure 3(e) & 17.43 & Figure 3(h) & 18.06 \\
Figure 3(c) & 11.52 & Figure 3(f) & 16.35 & Figure 3(i) & 17.10 \\
- & - & No. of & 5 & No. of & iterations \\
\end{tabular}




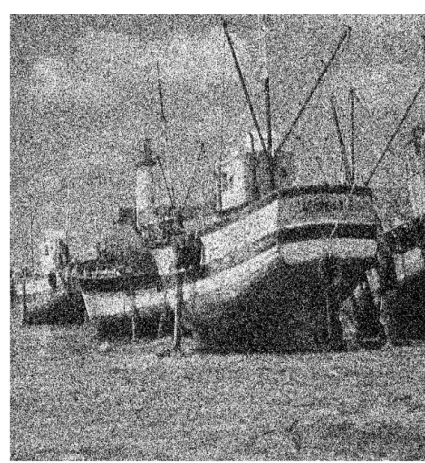

(a)

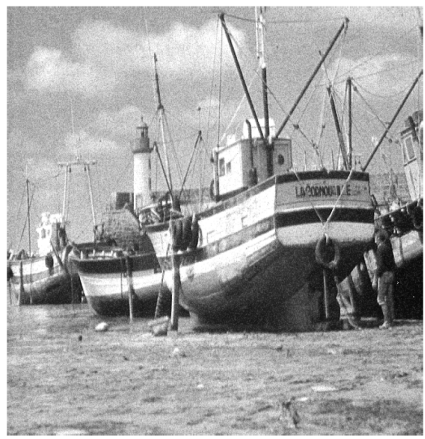

(d)

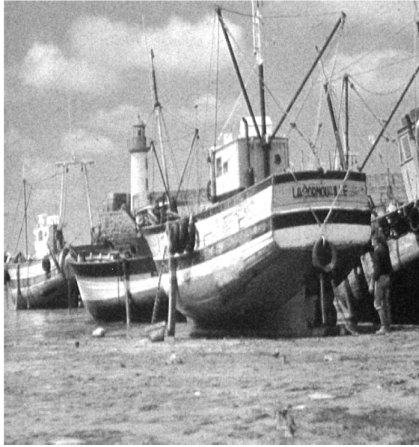

(g)

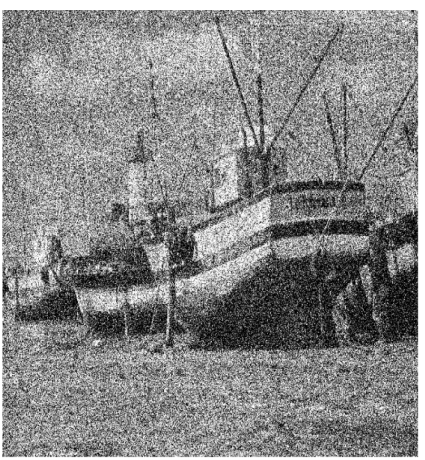

(b)

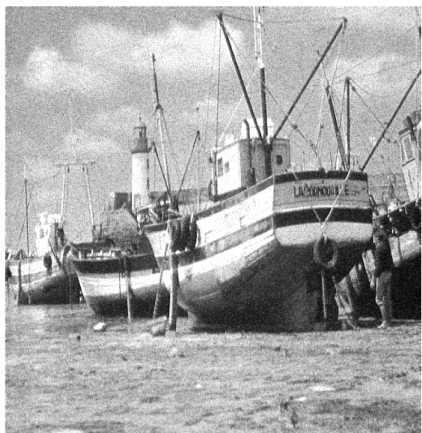

(e)

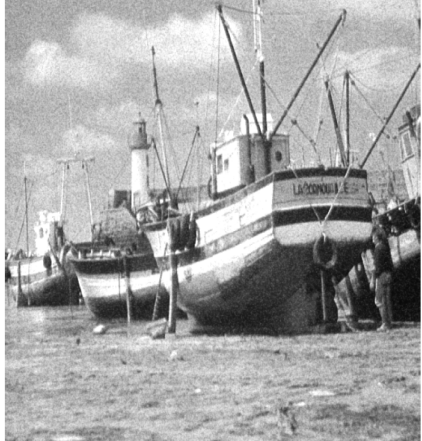

(h)

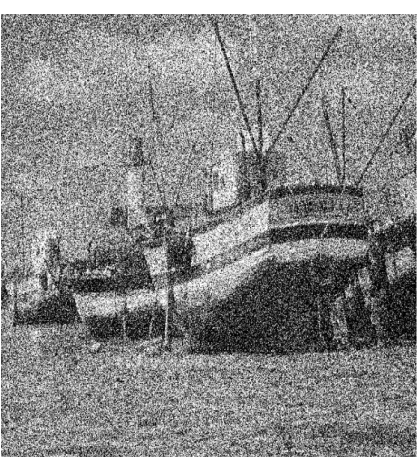

(c)

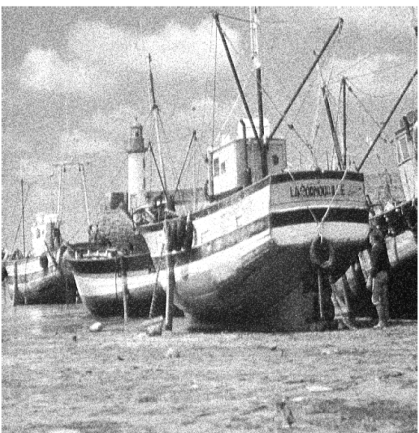

(f)

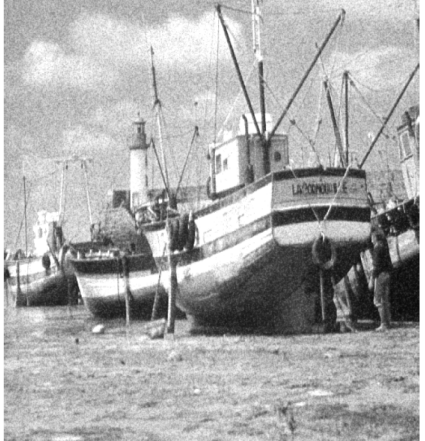

(i)

Figure 5. (Top row) Noisy Boat images with different levels of Gaussian noise (a)-(c); $\sigma^{2}=0.06,0.08,0.10$, respectively; (Second row) (d)-(f) corresponding denoised images by model (10); (Third row) (g)-(i) by model (11).

Table 2. Results obtained by using models (10) and (11) applied to the images in Figure 4 with three different levels of Gaussian noise $\left(\sigma^{2}=0.06,0.08\right.$ and 0.10$)$.

\begin{tabular}{cccccc}
\hline Images & PSNR & Images & PSNR & Images & PSNR \\
\hline & (Noisy Images) & & (Model-10) & & (Model-11) \\
\hline Figure 4(a) & 13.36 & Figure 4(d) & 19.10 & Figure 4(g) & 19.39 \\
Figure 4(b) & 12.38 & Figure 4(e) & 17.85 & Figure 4(h) & 18.26 \\
Figure 4(c) & 11.64 & Figure 4(f) & 16.86 & Figure 4(i) & 17.38 \\
- & - & No. of & 5 & No. of & 5 \\
& & iterations & & iterations & \\
\hline
\end{tabular}


Table 3. Results obtained by using models (10) and (11) applied to the images in Figure (5) with three different levels of Gaussian noise $\left(\sigma^{2}=0.06,0.08\right.$ and 0.10$)$.

\begin{tabular}{cccccc}
\hline Images & PSNR & Images & PSNR & Images & PSNR \\
\hline & (Noisy images) & & (Model-10) & & (Model-11) \\
\hline Figure 5(a) & 12.96 & Figure 5(d) & 17.07 & Figure 5(g) & 17.50 \\
Figure 5(b) & 11.97 & Figure 5(e) & 15.75 & Figure 5(h) & 16.28 \\
Figure 5(c) & 11.27 & Figure 5(f) & 14.73 & Figure 5(i) & No. of \\
- & - & No. of & 5 & iterations & 5 \\
\hline
\end{tabular}

used to discretize models (10) and (11). The model (11) gives larger PSNR values than that of model (10), at the same iteration numbers. Besides, a new time dependent model (22) to solve the signal denoising in 1D has also been given.

\section{References}

[1] Osher, S. and Rudin, L.I. (1990) Feature Oriented Image Enhancement Using Shock Filters. SIAM Journal on Numerical Analysis, 27, 919-940. http://dx.doi.org/10.1137/0727053

[2] Rudin, L., Osher, S. and Fatemi, E. (1992) Nonlinear Total Variation Based Noise Removal Algorithm. Physica D, 60, 259-268. http://dx.doi.org/10.1016/0167-2789(92)90242-F

[3] Rudin, L. and Osher, S. (1994) Total Variation Based Image Restoration with Free Local Constraints. IEEE International Conference on Image Processing, 1, 31-35.

[4] Chambolle, A. and Lions, P.L. (1997) Image Recovery via Total Variation Minimization and Related Problems. Numerische Mathematik, 76, 167-188. http://dx.doi.org/10.1007/s002110050258

[5] Hunt, B.R. (1973) The Application of Constrained Least Squares Estimation to Image Restoration by Digital Computer. IEEE Transactions on Computers, 22, 805-812. http://dx.doi.org/10.1109/TC.1973.5009169

[6] Strang, G. (1964) Accurate Partial Difference Methods II. Non Linear Problems. Numerische Mathematik, 6, 37-46. http://dx.doi.org/10.1007/BF01386051

[7] Strang, G. (1968) On the Construction and Comparison of Difference Schemes. SIAM Journal on Numerical Analysis, 5, 506-517. http://dx.doi.org/10.1137/0705041

[8] Phillips, D.L. (1962) A Technique for the Numerical Solution of Certain Integral Equations of the First Kind. Journal of the Association for Computing Machinery (ACM), 9, 84-97. http://dx.doi.org/10.1145/321105.321114

[9] Twomey, S. (1963) On the Numerical Solution of Fredholm Integral Equations of the First Kind by the Inversion of the Linear System Produced by Quadrature. Journal of the Association for Computing Machinery (ACM), 10, 97-101. http://dx.doi.org/10.1145/321150.321157

[10] Twomey, S. (1965) The Application of Numerical Filtering to the Solution of Integral Equations Encountered in Indirect Sensing Measurements. Journal of The Franklin Institute, 279, 95-109. http://dx.doi.org/10.1016/0016-0032(65)90209-7

[11] Chang, Q. and Chern, I.-L. (2003) Acceleration Methods for Total Variation-Based Image Denoising. SIAM Journal on Scientific Computing, 25, 982-994. http://dx.doi.org/10.1137/S106482750241534X

[12] Witkin, A.P. (1983) Scale-Space Filtering. International Joint Conference on Artificial Intelligence, 2, 1019-1021.

[13] Marquina, A. (2006) Inverse Scale Space Methods for Blind Deconvolution. UCLA CAM Report, 06-36.

[14] Alvarez, L., Lions, P.L. and Morel, J.M. (1992) Image Selective Smoothing and Edge Detection by Nonlinear Diffusion II. SIAM Journal on Numerical Analysis, 29, 845-866. http://dx.doi.org/10.1137/0729052

[15] Catte, F., Lions, P.L., Morel, J.M. and Coll, T. (1992) Image Selective Smoothing and Edge Detection by Nonlinear Diffusion. SIAM Journal on Numerical Analysis, 29, 182-193. http://dx.doi.org/10.1137/0729012

[16] Rosen, J.G. (1961) The Gradient Projection Method for Nonlinear Programming. Part II. Nonlinear Constraints. Journal of the Society for Industrial and Applied Mathematics, 9, 514-532. http://dx.doi.org/10.1137/0109044

[17] Lapidus, L. and Pinder, G.F. (1983) Numerical Solution of Partial Differential Equations in Science and Engineering. SIAM Review, 25, 581-582. http://dx.doi.org/10.1137/1025136 
[18] Marquina, A. and Osher, S. (2000) Explicit Algorithms for a New Time Dependent Model Based on Level Set Motion for Nonlinear Deblurring and Noise Removal. SIAM Journal on Scientific Computing, 22, 387-405. http://dx.doi.org/10.1137/S1064827599351751

[19] Chan, T.F., Golub, G.H. and Mulet, P. (1999) A Nonlinear Primal-Dual Method for Total Variation-Based Image Restoration. SIAM Journal on Scientific Computing, 20, 1964-1977. http://dx.doi.org/10.1137/S1064827596299767 
Scientific Research Publishing (SCIRP) is one of the largest Open Access journal publishers. It is currently publishing more than 200 open access, online, peer-reviewed journals covering a wide range of academic disciplines. SCIRP serves the worldwide academic communities and contributes to the progress and application of science with its publication.

Other selected journals from SCIRP are listed as below. Submit your manuscript to us via either submit@scirp.org or Online Submission Portal.
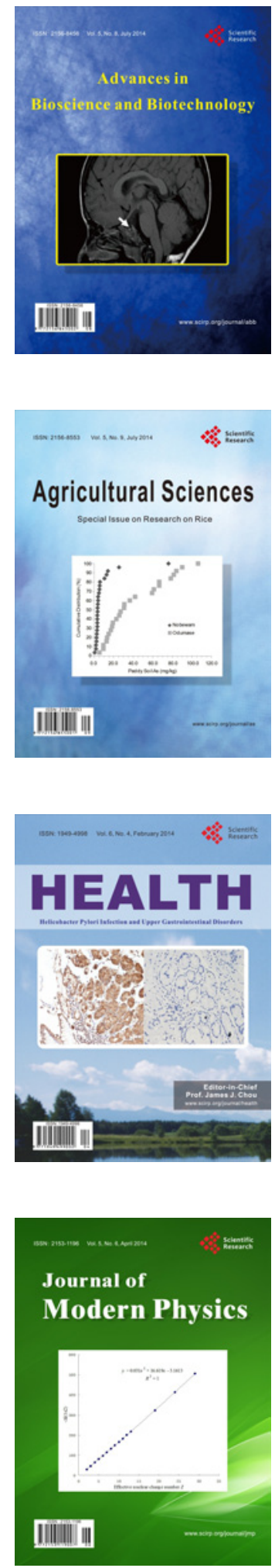
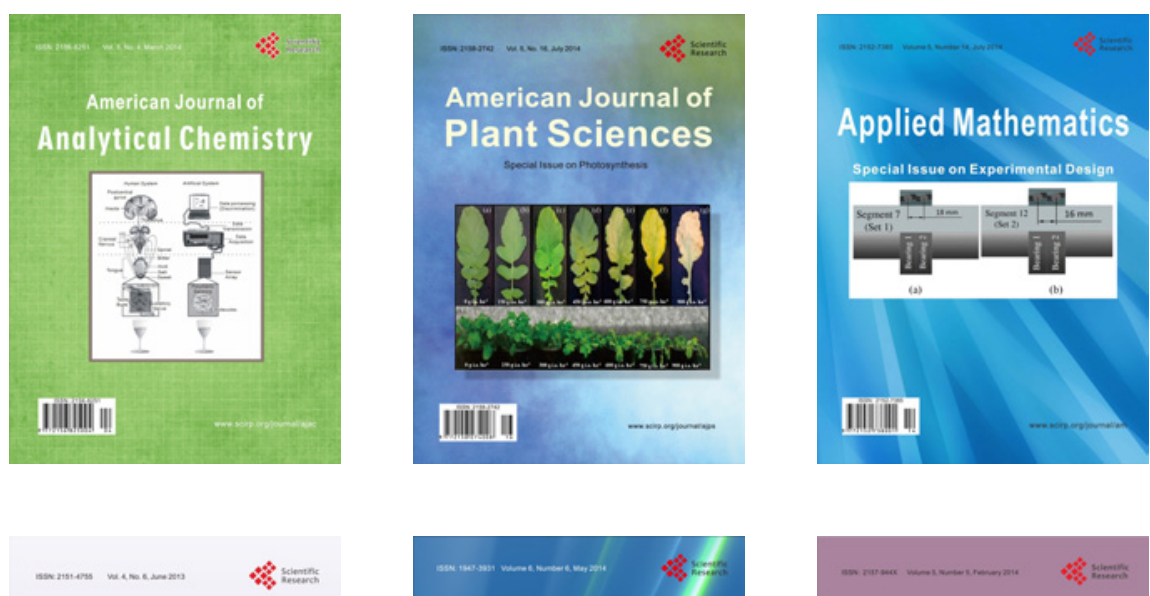

Creative Education
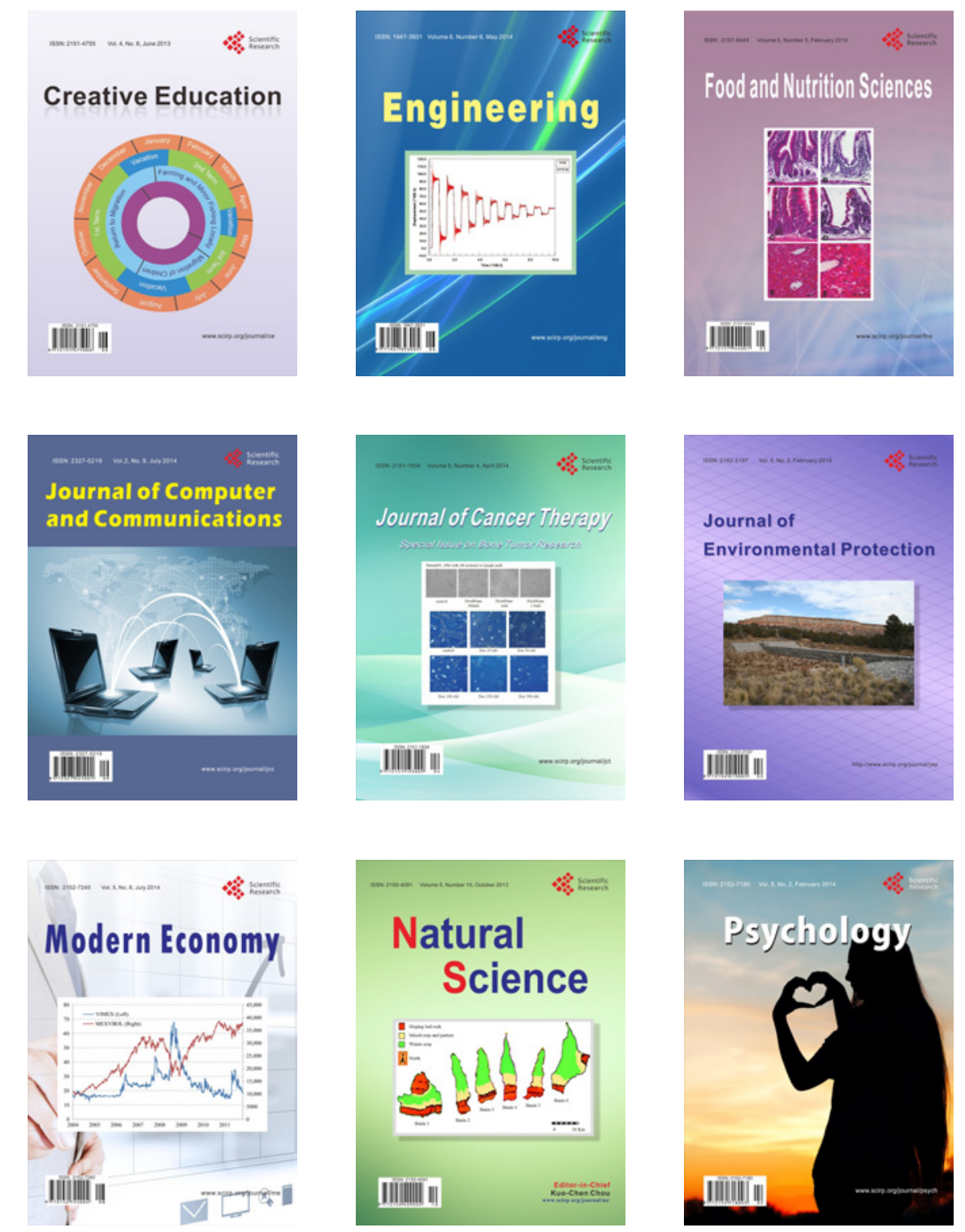